\title{
Comparative study between topical tranexamic acid and intradermal tranexamic acid injection in treatment of melasma
}

\section{Abdel Raheem TA ${ }^{(1)}$, Nassar MA ${ }^{(2)}$ and Elgamil MA ${ }^{(3)}$}

1- Professor of Dermatology, STDS and Andrology Faculty of Medicine, Fayoum University.

2- Lecturer of Dermatology, STDS and Andrology Faculty of Medicine, Fayoum University.

3-Department of Dermatology, Faculty of Medicine, Fayoum University.

Corresponding author : Prof . Talal A. Abd-ElRaheem

E-mail address: talasam @ yahoo.com

Tel: 01006600360

Fax : +2 084636583

\section{Abstract}

Melasma is an acquired hypermelanosis characterized by a symmetrical macular pigmentation of sun-exposed areas like the face. Tranexamic acid is commonly being used to reduce melanin synthesis in patients with melasma and also used as a raw material for functional whitening cosmetics. The aim of this work is to Assess efficacy of Tranexamic acid in the treatment of melasma. This study was conducted in Dermatology, STDS and Andrology Department of Fayoum University Hospital in the period between March 2016 and December 2016. Forty female patients, in the age group 25-50 years old, with different patterns of facial melasma, were included. They were treated with intradermal injection of Tranexamic acid in right side of the face and topical Tranexamic acid application after fractional carbon dioxide laser on the left side. All cases were assessed by modified Melasma Area and Severity Index score, photos before and after treatment and patient and physician satisfaction scoring. Our results shows Modified Melasma Area and Severity Index score of injected side decreased from $12 \pm 4.4$ to $9.6 \pm 3.8$ and modified Melasma Area and Severity Index score of laser side decreased from $11.8 \pm 4.8$ to $9.7 \pm 4.2$. There was no statistically significant difference with $p$-value $>0.05$ between two sides regarding patient and physician satisfaction denoting that Tranexamic acid is considered an effective treatment for melasma, there was no difference between intradermal injection of Tranexamic acid and usage of carbon dioxide laser as a route of delivery to topical Tranexamic acid. 
Keywords: Melasma- Tranexamic acid- Fractional carbon dioxide laser

\section{Introduction}

Melasma is a common acquired pigmentary skin disorder characterized by a symmetrical macular pigmentation of sun-exposed areas like the face[1]. Hypermelanosis of the skin is due to various underlying risk factors, including sun exposure, hormonal changes, pregnancy, genetic predisposition, combined oral contraceptives, use of cosmetics, photosensitizing drugs and inflammatory processes[2] .

Modified Melasma area and severity index (mMASI) was developed by Maluki and Al-Sabak, 2015 for the assessment of melasma. It would be useful in the measurement of the melasma before and after a treatment for each side of the face.

Tranexamic acid (TA) is a treatment option for melasma, which is being used orally, topically and intra dermally by microinjection or transepidermally by microneedling (mesotherapy) [3].TA is a plasmin inhibitor used to prevent abnormal fibrinolysis to reduce blood loss. It is a synthetic derivative of the amino acid lysine and exerts its effect by reversibly blocking lysine binding sites on plasminogen molecules, thus inhibiting plasminogen activator (PA) from converting plasminogen to plasmin [4].

TA is one of the treatment strategies for melasma that has been a focus of attention in recent years. Potential mechanisms for its effectiveness include the following: inhibition of melanocyte proliferation, inhibition of melanin synthesis in melanocytes, reduced number of blood vessels in the dermis and reduced number of mast cells in the dermis [5]

Strategies to enhance transdermal delivery of TA have been developed. Fractional carbon dioxide $\left(\mathrm{CO}_{2}\right)$ laser was reported to increase TA absorption [6] . Laser-assisted drug delivery (LADD) is promising future approach to treat melasma[7].

\section{Patients and method}

aim of this study is to Assess efficacy of Tranexamic acid in the treatment of melasma. This study was conducted on Forty female patients with melasma subjected to examination by general and local examination and dermoscopy to 
determine type of melasma. Then the patients had done split face therapy. Right side of the face was treated with intradermal injection of TA, and the left side was treated with fractional $\mathrm{CO}_{2}$ laser followed by application of TA topically in three sessions by one month interval. Photographic records were obtained pretreatment, after every session and after the end of the treatment. 


\section{Results}

1.This study revealed that there was highly statistically significant difference with $p$-value $<0.05$ in mean mMASI score after treatment in each side and significant decrease in mMASI score after treatment in both procedures, which indicats that both procedures improved melasma .

\begin{tabular}{|c|c|c|c|c|}
\hline \multirow[t]{2}{*}{ mMASI score } & \multicolumn{2}{|c|}{$\begin{array}{c}\text { Laser } \\
\text { procedure } \\
(n=40)\end{array}$} & \multicolumn{2}{|c|}{$\begin{array}{l}\text { Injection } \\
\text { procedure } \\
\quad(n=40)\end{array}$} \\
\hline & Mean & SD & Mean & SD \\
\hline Before treatment & 11.8 & 4.8 & 12 & 4.4 \\
\hline After treatment & 9.7 & 4.2 & 9.6 & 3.8 \\
\hline$p$-value & \multicolumn{2}{|c|}{$<0.001$} & \multicolumn{2}{|c|}{$<0.001$} \\
\hline Sig. & \multicolumn{2}{|c|}{ HS } & \multicolumn{2}{|c|}{ HS } \\
\hline
\end{tabular}

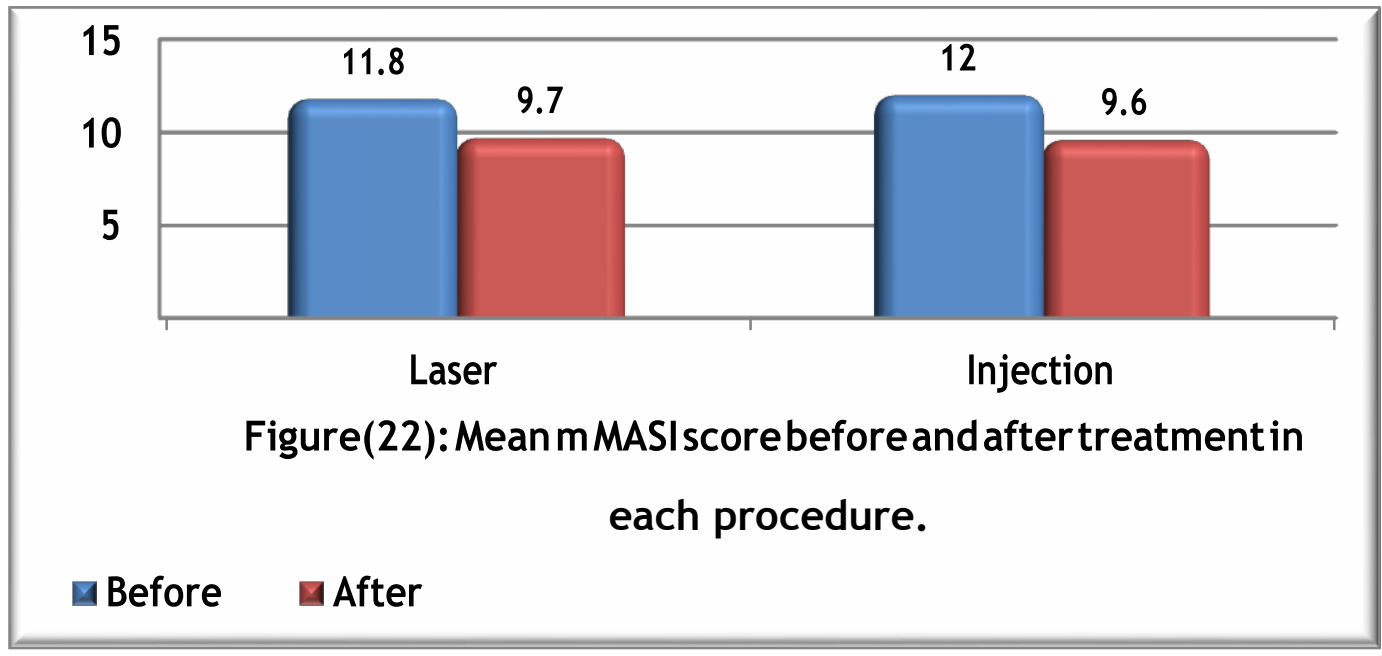

2.There was no statistically significant difference with $p$-value $>0.05$ between different study procedure in mean mMASI score before and after treatment, which indicated that both procedures nearly have the same effect on the patients . 


\begin{tabular}{|c|c|c|c|c|c|c|}
\hline \multirow[t]{2}{*}{ mMASI score } & \multicolumn{2}{|c|}{$\begin{array}{c}\text { Laser } \\
\text { procedure } \\
(\mathrm{n}=40)\end{array}$} & \multicolumn{2}{|c|}{$\begin{array}{c}\text { Injection } \\
\text { procedure } \\
(\mathrm{n}=40)\end{array}$} & \multirow[t]{2}{*}{$p$-value } & \multirow{2}{*}{ Sig. } \\
\hline & Mean & SD & Mean & SD & & \\
\hline Before treatmet & 11.8 & 4.8 & 12 & 4.4 & 0.8 & NS \\
\hline After treatmet & 9.7 & 4.2 & 9.6 & 3.8 & 0.9 & NS \\
\hline
\end{tabular}

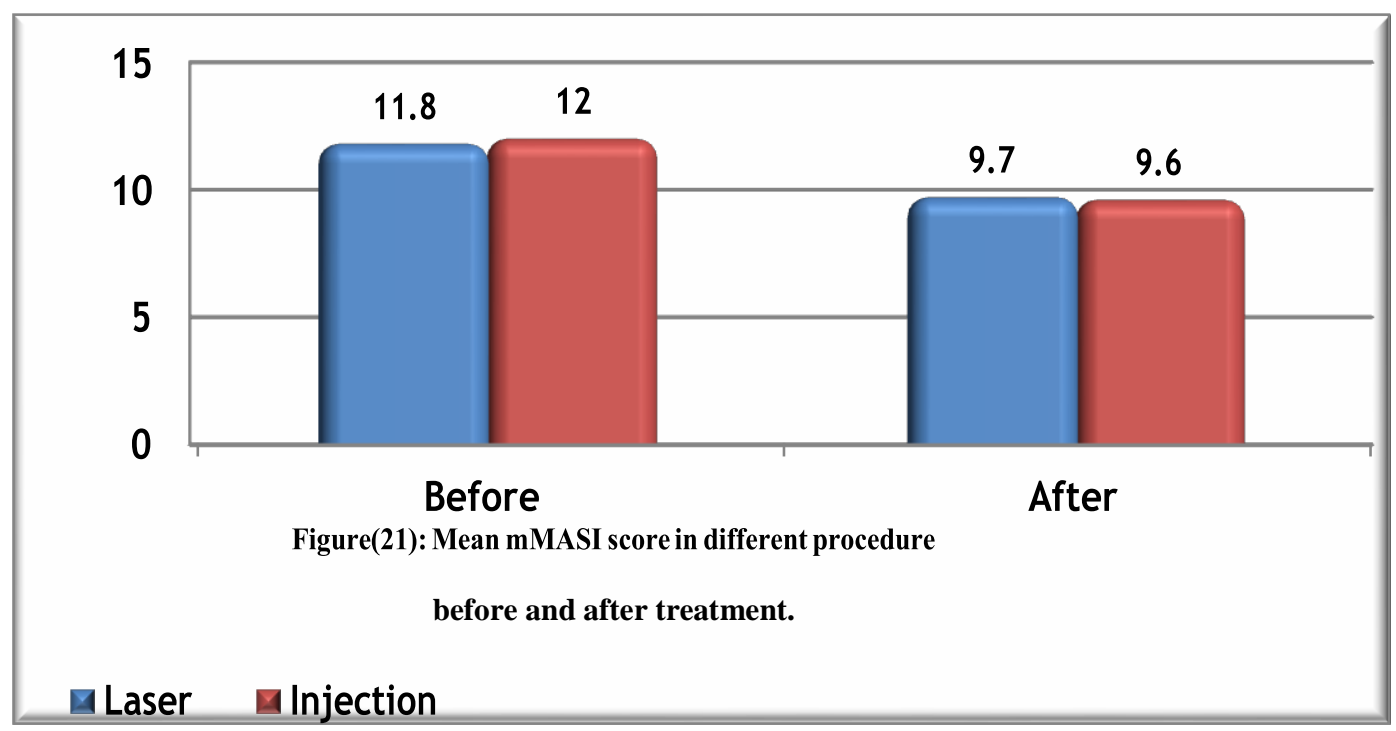

3. This study revealed that there was significant difference with $p$-value $<0.05$ in mMASI score before and after treatment between different types of melasma with high difference between mean of mMASI score before and after treatment among dermal type of melasma

\begin{tabular}{||c|c|c|c|c||}
\hline \multirow{2}{*}{ Types of melasma } & \multicolumn{4}{|c||}{ mMASI score } \\
\cline { 2 - 6 } & \multicolumn{2}{|c|}{ Before } & \multicolumn{2}{c||}{ After } \\
\cline { 2 - 6 } Epidermal & Mean & SD & Mean & SD \\
\hline Dermal & 11.7 & 4 & 9.5 & 3.8 \\
\hline Mixed & 14.1 & 6.3 & $\mathbf{1 1 . 5}$ & 4.7 \\
\hline$p$-value & 10.2 & 2.7 & 8 & 2.9 \\
\hline Sig. & \multicolumn{2}{|c|}{$\mathbf{0 . 0 2}$} & \multicolumn{2}{c|}{$\mathbf{0 . 0 2}$} \\
\hline
\end{tabular}




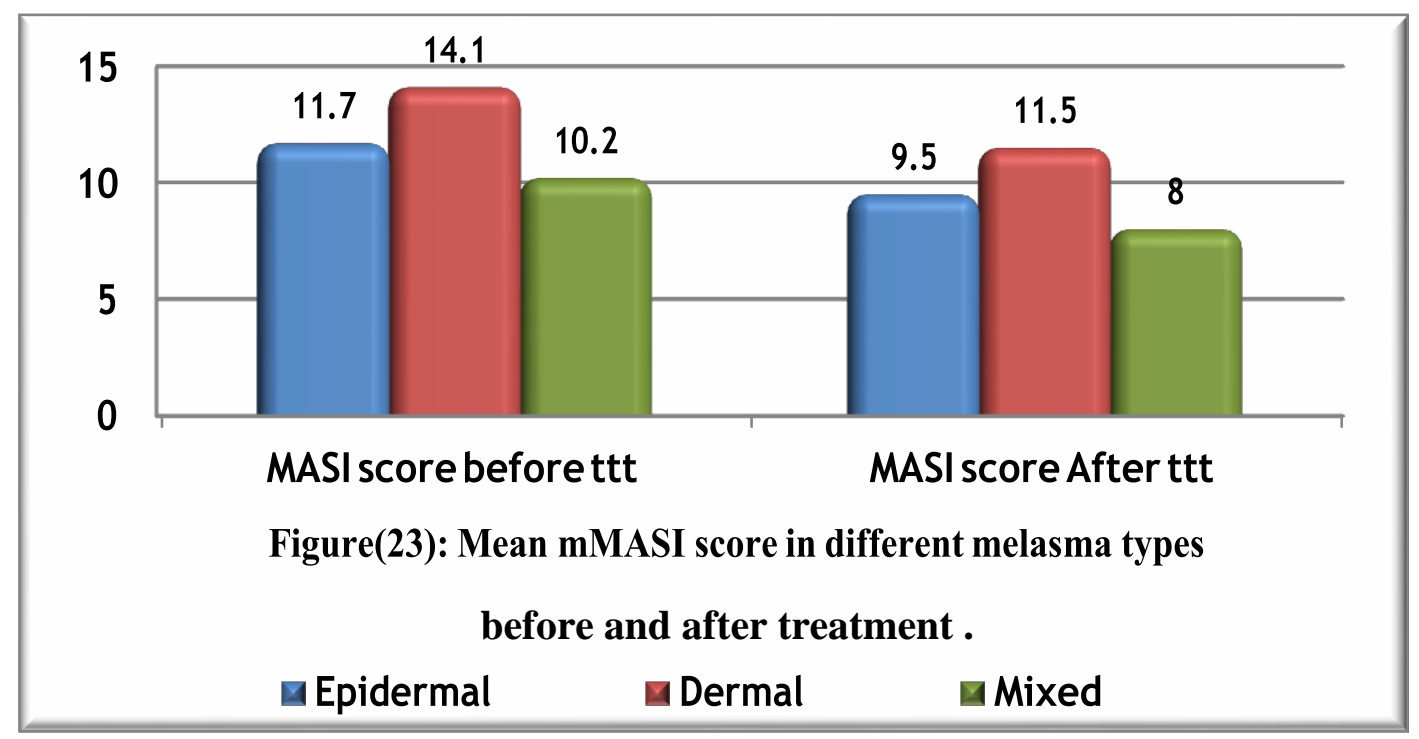

4.When studying the Correlation between mMASI score in relation to age and Disease duration There was statistically significant positive correlation with $p$-value $<0.05$ between m MASI score before and after treatment with age and duration of disease, which indicated that increasing in age and disease duration was associated with increasing in mMASI score before and after treatment

\begin{tabular}{|c|c|c|c|c|c|c|}
\hline \multirow{2}{*}{ Variables } & \multicolumn{3}{|c|}{ mMASI score before } & \multicolumn{2}{l|}{ mMASI score after } \\
\cline { 2 - 7 } & r & $p$-value & Sig. & r & $p$-value & Sig. \\
\hline Age (years) & 0.37 & $\mathbf{0 . 0 0 1}$ & HS & 0.38 & $<0.001$ & HS \\
\hline $\begin{array}{c}\text { Disease duration } \\
\text { (years) }\end{array}$ & 0.26 & $\mathbf{0 . 0 2}$ & $\mathbf{S}$ & 0.30 & $\mathbf{0 . 0 0 7}$ & HS \\
\hline
\end{tabular}




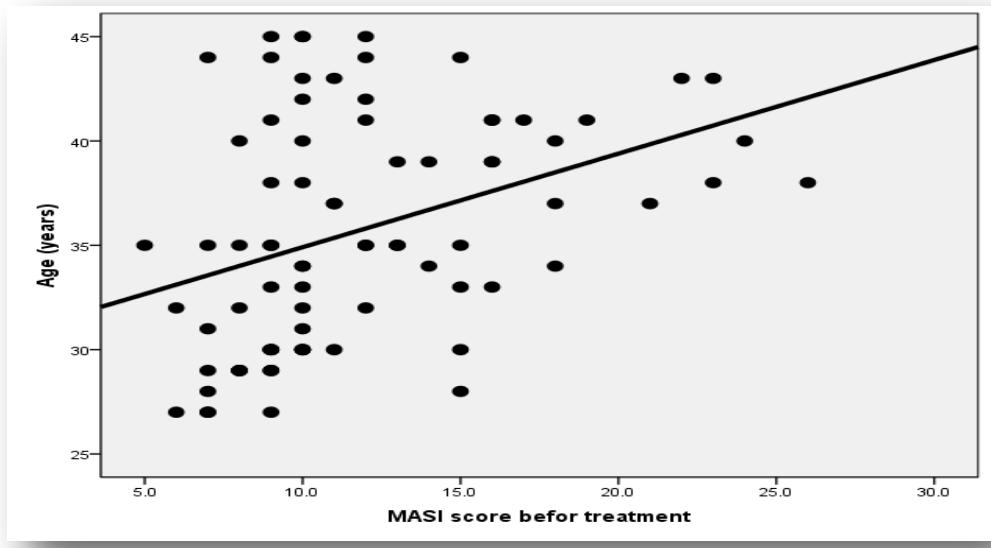

\section{Discussion}

Melasma is a dysregulation of the homeostatic mechanisms that control skin pigmentation and excess pigment is produced. Traditional treatment approaches with topical medications and chemical peels are commonly used but due to the refractory and recurrent nature of melasma, patients often seek alternative treatment strategies (Trivedi et al., 2017).

TA is a treatment option for melasma; it may inhibit melanin synthesis by blocking the interaction between melanocytes and keratinocytes and reverse the abnormal dermal changes associated with melasma, such as the increased vasculature ( $\mathrm{Na}$ et al., 2013).In this study, the mean mMASI score before treatment was $(12 \pm 4.4)$ versus (9.6 \pm 3.8$)$ after treatment in injection procedure. There was highly significance decrease in mMASI score from the base line to the end of the treatment. These results were in agreement with Lee et al., (2006) , Steiner et al., (2009), Budamakuntla et al., (2013) and Elfar and El-Maghraby, (2015).

In this study mMASI before treatment with fractional $\mathrm{CO} 2$ laser and topical TA side was $11.8 \pm 4.8$ versus $9.7 \pm 4.2$ after treatment. Studies used topical TA Banihashemi et al., (2015) MASI improved from $14.72 \pm 2.2$ to $7.69 \pm 2.4$, Atefi et al., (2017) MASI improved from $4.80 \pm 1.06$ to $2.33 \pm 0.71$ and Budamakuntla et al., (2013) used the micro needling to increase absorption of topical TA; the mean MASI score at the baseline was $9.11 \pm 4.09$ and at the end of third follow-up was $5.06 \pm 2.14$. In this 
study there was no statistically significant difference with $p$-value $>0.05$ between intradermal injected TA and with topical TA sides in mean mMASI score before and after treatment, These results were in agreement with Budamakuntla et al., (2013)which compared intradermal injection of TA and micro needling with topical TA and there was no significant difference in the means of the MASI scores between the intradermal injection and micro needling group.

In this study there was significant difference with $p$-value $<0.05$ in mMASI score before and after treatment between different types of melasma with high difference between mean of mMASI score before and after treatment among dermal type of melasma. This indicates that both procedures may improve dermal type of melasma which is always difficult to treat as it is out of rich to topical drugs. So that combinations of these procedures with traditional treatments of melasma may give promising results.

\section{Conclusion}

In our study we concluded that:

Our findings suggest that TA can play a role in melasma treatment.

*TA intradermal injection or topical TA with laser assisted technique can be used as potentially a safe and effective therapeutic agent for treatment of melasma.

*Both methods can be effective in treatment of dermal type which is always difficult in treatment.
*Both intradermal injection TA and fractional $\mathrm{CO}_{2}$ assisted drug delivery had nearly the same effect on melisma.

In this study there was significant difference with $p$-value $<0.05$ in mMASI score before and after treatment between different types of melasma with high difference between mean of mMASI score before and after treatment among dermal type of melasma. This indicates that both procedures may improve dermal type of melasma which is always difficult to treat as it is out of rich to topical drugs. So that combinations of these procedures with traditional treatments of melasma may give promising results. 


\section{Conclusion}

In our study we concluded that:

Our findings suggest that TA can play a role in melasma treatment.

*TA intradermal injection or topical TA with laser assisted technique can be used as potentially a safe and effective therapeutic agent for treatment of melasma.

*Both methods can be effective in treatment of dermal type which is always difficult in treatment.

*Both intradermal injection TA and fractional $\mathrm{CO}_{2}$ assisted drug delivery had nearly the same effect on melisma.

\section{References}

- 1. Sarkar R, Gokhale N, Godse K, Ailawadi P, Arya L, Sarma N, Torsekar RG, Somani VK, Arora P, Majid I, Ravichandran G, Singh M, Aurangabadkar S, Arsiwala S, Sonthalia S, Salim T and Ravichandran G (2017): Medical management of melasma: A review with consensus recommendations by Indian pigmentary expert group. Indian journal of dermatology; 62 (6), 558-577.

- 2. Atefi N, Dalvand B, Ghassemi M, Mehran G and Heydarian A (2017): Therapeutic Effects of Topical Tranexamic Acid in Comparison with Hydroquinone in Treatment of Women with Melasma. Dermatology and therapy; 7(3), 417-424.

- 3. Budamakuntla L, Loganathan E, Suresh DH, Shanmugam

S, Suryanarayan

S, Dongare

A, Venkataramiah LD and Prabhu $N$ (2013): A randomised, open-label, comparative study of tranexamic acid microinjections and tranexamic acid with microneedling in patients with melasma. Journal of cutaneous and aesthetic surgery; 6(3), 139-143.

- 4.Tse TW and Hui E (2013): Tranexamic acid: an important adjuvant in the treatment of melasma. Journal of cosmetic dermatology; 12(1), 57-66.

- 5. XuY, Ma R, Juliandri J, Wang X, Xu B, Wang D, Lu Y, Zhou B and Luo D (2017): Efficacy of functional microarray of microneedles combined with topical tranexamic acid for melasma: A randomized, self-controlled, split-face study. Medicine; 96(19), 6897.

- 6. Hsiao CY, Sung HC, Hu S and Huang CH (2015): Fractional CO2 laser treatment 
to enhance skin permeation of tranexamic acid with minimal skin disruption. Dermatology; 230(3), 269-275.

- 7. Trivedi MK, Yang FC and Cho BK (2017):

A review of laser and light therapy in melasma.
International Journal of Women's Dermatology; 3(1):11-2. 